\title{
Minority stress, resilience, and health in Italian and Taiwanese LGB+ people: A cross-cultural comparison
}

\author{
Roberto Baiocco ${ }^{1}$ (D) - Cristiano Scandurra ${ }^{2}$ (D) Fausta Rosati $^{1}$ (D) Jessica Pistella ${ }^{1}$ (D) Salvatore loverno ${ }^{3}$ (D) \\ Vincenzo Bochicchio ${ }^{4}$ (D) Hung-Che Wang ${ }^{5}$ (D) Te-Sheng Chang $^{5}$ (D)
}

Accepted: 14 January 2021 / Published online: 27 January 2021

(C) The Author(s) 2021

\begin{abstract}
The present study, using a moderated mediational model, explored levels of distal/proximal stressors, rumination, resilience, and health in a group of Italian and Taiwanese LGB+ people. The study also examined the role of internalized sexual stigma (ISS) and rumination as mediators between discrimination and health, and resilience as a moderator of the relationship between discrimination and ISS, rumination, and health, respectively. An online survey was administered to 508 LGB+ participants (270 Italian and 238 Taiwanese) whose age ranged from 18 to 70 years $(M=37.93, S D=13.53)$. The moderated mediation model was tested through a series of path analyses stratified by group nationality. Italian participants reported higher discrimination and resilience, but lower ISS, rumination, and health problems compared to their Taiwanese counterparts. The only common path between groups was the direct effect of discrimination on health problems. The mediating role of ISS and rumination in the relationship between discrimination and health, as well as the moderating role of resilience, were partly significant only for the Italian group. Conclusions: The findings suggest that mediators and moderators used to evaluate the effects of minority stress on health may differ between groups; further culturally sensitive research in the field of LGB+ health is needed.
\end{abstract}

Keywords Minority stress $\cdot$ Health $\cdot$ LGB $+\cdot$ Italian $\cdot$ Taiwanese

Lesbian, gay, bisexual, and other sexual minority (LGB+) people are at higher risk of developing several negative health outcomes (e.g., substance abuse, depression, anxiety, etc.) compared to their heterosexual counterparts (Bostwick, Boyd, Hughes, West, \& McCabe, 2014; Gonzales \& Henning-Smith, 2017; Potter \& Patterson, 2019). These health disparities may be due to the systematic discrimination and oppression experienced by LGB+ people, as members of a strongly stigmatized population (Hässler et al., 2020; Hatzenbuehler, 2010; Russell \& Fish, 2016). The predominant theoretical frameworks that are used to understand how social stigma affects the health of LGB+ people are minority
Roberto Baiocco

roberto.baiocco@uniroma1.it

Cristiano Scandurra

cristiano.scandurra@unina.it

Fausta Rosati

fausta.rosati@uniroma1.it

Jessica Pistella

jessica.pistella@uniroma1.it

Salvatore Ioverno

salvatore.ioverno@ugent.be

Vincenzo Bochicchio

vincenzo.bochicchio@unical.it

Hung-Che Wang

strewang@gmail.com
Te-Sheng Chang

achang@gms.ndhu.edu.tw

1 Department of Developmental and Social Psychology, Faculty of Medicine and Psychology, Sapienza University of Rome, Rome, Italy

2 Department of Neuroscience, Reproductive Sciences and Dentistry, University of Naples Federico II, Naples, Italy

3 Department of Sociology, Ghent University, Ghent, Belgium

4 Department of Humanistic Studies, University of Calabria, Rende, CS, Italy

5 Department of Education and Human Potentials Development, National Dong Hwa University, Hualien, Taiwan 
stress theory (MST; Meyer, 2003) and the psychological mediation framework (PMF; Hatzenbuehler, 2009); the latter may be considered a clinical extension of the former.

MST posits that social stigma originates within stigmatizing social environments, resulting in unique and chronic stress that increases subjects' risk of developing negative health outcomes (Meyer, 2003). Furthermore, MST postulates the existence of two types of stressors: (a) objective stressors (i.e., distal stressors, such as discrimination) and (b) subjective stressors (i.e., proximal stressors, such as internalized sexual stigma [ISS], which describes self-identification with negative social values). Amongst LGB+ people, the combination of distal and proximal stressors increases the risk of developing health problems (Denton, Rostosky, \& Danner, 2014; Douglass \& Conlin, 2020; Toomey, Ryan, Diaz, \& Russell, 2018; Walch, Ngamake, Bovornusvakool, \& Walker, 2016).

However, MST attributes a fundamental role to resilience as a protective factor ameliorating the detrimental effects of minority stress (MS) on health (Meyer, 2015). Resilience may be defined as a dynamic psychological process embracing positive adaption within contexts of significant adversity and as an ability to negotiate with social contexts generating access to necessary resources in order to overcome difficulties (Luthar, Cicchetti, \& Becker, 2000). LGB+ individuals may benefit from both individual and group/community level resilience factors (de Lira \& de Morais, 2018; Hill \& Gunderson, 2015; Kwon, 2013; Matsuno \& Israel, 2018; Meyer, 2015). Specifically, individual-level resilience factors refer to subjective qualities that $\mathrm{LGB}+$ people may use to develop resilience in the face of stress (e.g., personal agency, self-worth, personality characteristics). In contrast, group/community-level resilience factors refer to how groups or communities provide resources helping individuals cope with stress and stigma, emphasizing the environmental influences on health (e.g., positive role models, community belonging, family acceptance). To date, there is significant evidence that both individual and group/community resilience factors are effective in ameliorating the adverse effects of minority stressors on health, depicting a resilient population able to use adaptive strategies in adverse contexts (Baiocco, Pezzella, Pistella, et al., 2021; Bariola, Lyons, \& Lucke, 2017; Jackson, 2017; Riggle, Whitman, Olson, Rostosky, \& Strong, 2008).

PMF contributes a more clinical view of stress, highlighting the effect of stigma-related stressors on health (Douglass \& Conlin, 2020; Kiekens et al., 2020). The framework postulates that distal stressors may impact health through the mediating action of both group-specific processes (i.e., proximal stressors, such as ISS) and general psychological processes (e.g., rumination, which describes repetitive self-focus on one's own symptoms and negative feelings, and their causes and consequences; Timmins, Rimes, \& Rahman, 2020). To this end, ISS may be considered the most insidious subjective minority stressor (Meyer, 2003), associated with many negative health outcomes (Berg, Munthe-Kaas, \& Ross, 2016; Salvati, Pistella, Ioverno, Laghi, \& Baiocco, 2018). Similarly, as rumination represents a maladaptive emotion regulation strategy for responding to general life stressors (McLaughlin \& Hatzenbuehler, 2009), it is important to determine whether and how LGB+ people may use it to manage their chronic stress (Hatzenbuehler, 2009). Indeed, in samples of LGB+ individuals, rumination has been found to be associated with increased distress (Hatzenbuehler, Dovidio, Nolen-Hoeksema, \& Phills, 2009; Lewis, Milletich, Mason, \& Derlega, 2014; Szymanski, Dunn, \& Ikizler, 2014).

Despite such evidence, cross-cultural studies of the role of social context in predicting differences in MS and health in LGB+ populations are scarce. Notably, a recent study analyzing the cross-cultural robustness of MST in Western European, Eastern European, Indian, Filipino, and Thai gay and bisexual men found it to be a good cross-cultural model for explaining satisfaction with life in this population (Sattler \& Lemke, 2019). Notwithstanding, no previous study has compared MST variables in Italian and Taiwanese LGB+ individuals.

To this end, although the Italian society seems increasingly accepting of LGB+ individuals (Lingiardi et al., 2016), many recent Italian studies have detected moderate levels of discrimination against this population (Baiocco \& Pistella, 2019; Petrocchi et al., 2020; Picariello, Scandurra, Schwartz, \& Amodeo, 2019; Scandurra et al., 2020). In Italy, LGB people still face heterosexism in several contexts, such as school, sports, and family: No current legislation to protect people from homophobic harassment and discrimination, and there is little or no visibility of $\mathrm{LGB}+$ people and relationships in many institutions (Baiocco \& Pistella, 2019). However, the discrimination experienced by the $\mathrm{LGB}+$ population may have stimulated them to create communities to oppose stigma and develop resilience skills and fighting for equality and the rights of the modern LGBT+ movement (Baiocco et al., 2021).

People in Taiwan traditionally regard sexual minorities as a challenge to the family obligations mandated in Confucianism, and they require their children to continue the family bloodline (Lin et al., 2020). However, in the past two decades, social tolerance has become widespread in Taiwan with an improvement in education and liberal values related to homosexuality and gender roles. Studies of the LGB+ population in Taiwan are scarce; however, similar to studies in the Italian context, recent Taiwanese studies have not depicted a favorable situation for LGB+ individuals (Hu, Chang, Lin, \& Yen, 2019; Huang, Chan, \& Cui, 2020a; Huang, Luo, Ko, \& Yen, 2020b), even though Taiwan was the first Asian country to introduce samesex marriage through voter-initiated referendums, and allowed for same-sex second-parent adoption (2019).

Intercultural differences in the associations between MST dimensions would be expected between these national contexts, as Western and Eastern cultures are significantly 
differentiated according to their individualistic versus collective attitudes, respectively (Sattler \& Lemke, 2019). Individualistic societies prioritize the needs of an individual over the needs of a group as a whole. People in a collectivist culture tend to see themselves as interdependent within their groups and usually behave according to collectivist social norms (Liu et al., 2011). People in a collectivist society are advised to exercise emotional restraint to avoid shame and rejection. In contrast, individuals in an individualist culture see themselves as independent of groups and generally behave according to personal choices. Taiwanese LGB+ people, influenced by a collectivist culture, may tend to subordinate their personal interests to those of the group or collective (Liu et al., 2011). A collectivist society devalues individuals who are considered nonconforming to group values. According to Confucianism, individuals are defined within the context of their familial relationships. The cultural imperative of familial responsibility results in the stigmatization of $\mathrm{LGB}+$ people, and it is generally associated with poor health and a high level of ISS (Liu et al., 2011).

The present study aimed at applying MST and the PMF to explore potential differences between Italian and Taiwanese LGB+ participants. Specifically, we aimed to examine levels of distal/proximal stressors, rumination, resilience, and health in the two subgroups using a moderated mediational model. The study also examined the role of ISS and rumination as mediators between discrimination and health, and resilience as a moderator of the relationship between discrimination and ISS, rumination, and health, respectively. Based on the aforementioned theories and empirical findings, we made several predictions. First, based on MST, we hypothesized that discrimination (i.e., a distal stressor) would be positively associated with health problems (Hypothesis 1). Second, based on the PMF, we hypothesized that ISS (i.e., a proximal stressor) and rumination (i.e., a general psychological process) would mediate the relationship between discrimination and health (Hypothesis 2), and that resilience would moderate the relationship between discrimination and ISS, rumination, and health, respectively (Hypothesis 3). Finally, we hypothesized that the indirect effect of discrimination on health problems through ISS and rumination would be moderated by resilience (Hypothesis 4). We had no specific hypotheses regarding the effect of each country on health disparities. For all the hypotheses, potential differences between countries were considered. The hypothesized moderated mediation model is depicted in Fig. 1.

\section{Method}

\section{Procedure and Participants}

The present study administered a cross-sectional online survey via the Unipark software. The survey was distributed through Italian and Taiwanese LGB+ social network pages and groups between January and November 2019. Specifically, respondents were recruited through online advertisements and an online-based survey. For each country, the online advertisements were posted: (a) on the main home page of LGB+ associations; (b) on the home page and social network pages of the specific institutions of the partners involved in the project (University of XXX in Italy and University of XXX in Taiwan). Again, participants were recruited via electronic mail and professional mailing lists from university and community settings. Stakeholders and representatives of LGB+ organizations were contacted directly and asked to share the survey with their networks, activating a snowball sampling procedure. Participation was voluntary and anonymous. The inclusion criteria were: (a) self-identifying as LGB+; (b) being 18 years or older; and (c) being Italian or Taiwanese. Participation was voluntary and anonymous, and all respondents answered the same set of questionnaires that required approximately 25-30 min to complete. Informed consent was required on the first page of the survey, where information about the research project was presented. The study was designed in accordance with the Declaration of Helsinki and approved by the Ethical Committee of the XXXX University of XXXX. Data are available upon request in compliance with the General Data Protection Regulation (GDPR; Regulation EU2016/679) on the protection of natural persons with regard to the processing of personal data and on the free movement of such data.

Participants were $508 \mathrm{LGB}+$ people (of whom 270 were Italian) whose age ranged from 18 to 70 years $(M=37.93$, $S D=13.53)$. Full socio-demographic characteristics are presented in Table 1. Italian and Taiwanese LGB+ people showed significant differences in age, biological sex, gender identity, and educational level, but not sexual orientation and socioeconomic status (SES). Specifically, Taiwanese participants were younger, more educated, and less balanced in terms of gender (with a higher proportion of men) than the Italian group.

\section{Measures}

Socio-Demographic Information Socio-demographic variables included age, nationality, biological sex $(0=$ female, $1=$ male $)$, gender identity $(0=$ men, $1=$ women, $3=$ other, indicate), sexual orientation $(0=$ lesbian/gay, $1=$ bisexual/other), educational level ( $0=\leq$ high school, $1=\geq$ college $)$, and SES ( 0 = low-medium, 1 = high $)$.

Discrimination Discrimination was assessed using the Discrimination/Harassment subscale of the Daily Heterosexist Experiences Questionnaire (Balsam, Beadnell, \& Molina, 2013). This measure is a 50-item scale measuring LGB+ people's daily minority stressors stemming from societal oppression. This subscale is composed of six items, 
Fig. 1 The Hypothesized Moderated-Mediation Model

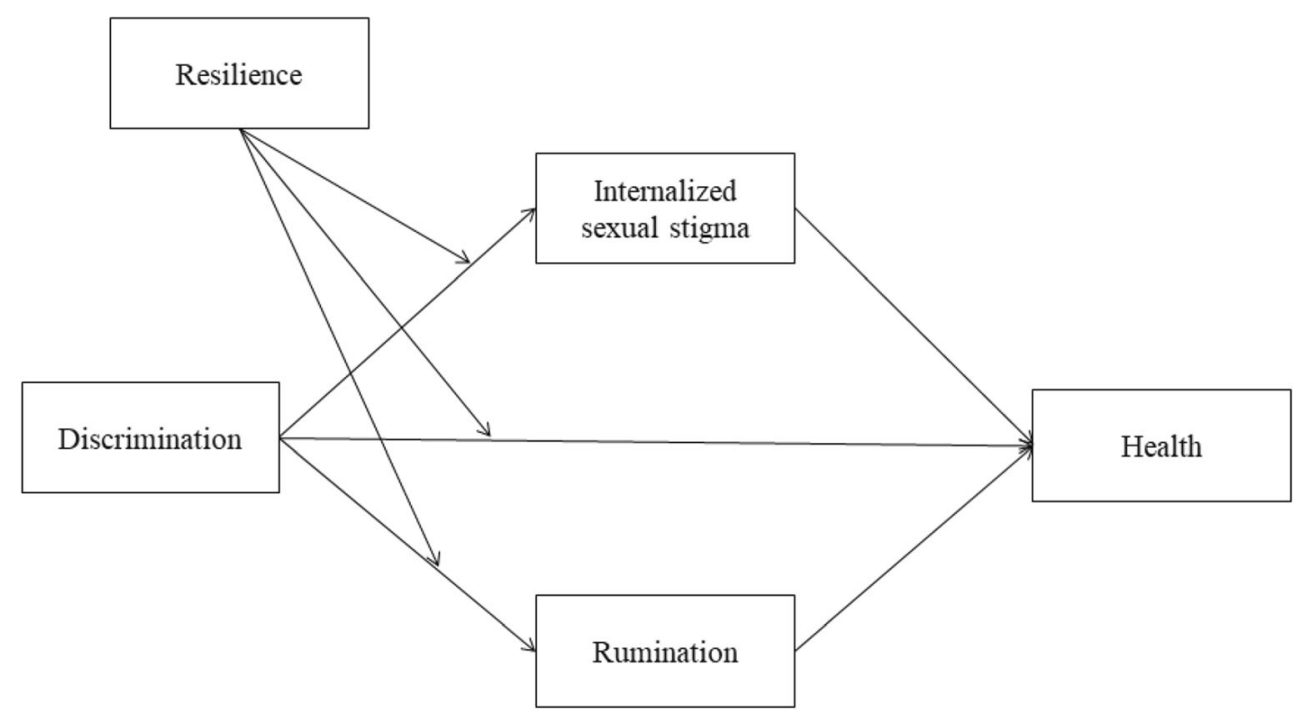

ranging from 0 (this did not bother me at all) to 4 (this bothered me extremely), designed to capture the salience of discriminatory experiences (i.e., "Being verbally harassed by strangers because you are LGBT"). Each item is also dichotomized ( $0=\operatorname{did}$ not happen; $1=$ happened) with higher scores indicating a higher frequency of discrimination $(\alpha=0.91)$.

Internalized Sexual Stigma ISS was measured using the short version of the Measure of Internalized Sexual Stigma for
Lesbians and Gay Men (Lingiardi, Baiocco, \& Nardelli, 2012), which is a six-item scale assessing sexual minorities' negative attitudes toward themselves (i.e., "I do not believe in love between LGB+ people"). Answers range from 1 ( disagree) to 5 (I agree), with higher scores indicating greater ISS $(\alpha=0.90)$.

Rumination Rumination was assessed using the short version of the Ruminative Response Scale (Treynor, Gonzalez, \&
Table 1 Socio-Demographic Characteristics of Participants $(n=508)$

\begin{tabular}{|c|c|c|c|c|}
\hline Characteristics & $\begin{array}{l}\text { Total } \\
(n=508) \\
N(\%) \text { or } M \pm S D\end{array}$ & $\begin{array}{l}\text { Italian } \\
(n=270) \\
N(\%) \text { or } M \pm S D\end{array}$ & $\begin{array}{l}\text { Taiwanese } \\
(n=238) \\
N(\%) \text { or } M \pm S D\end{array}$ & $t / \chi^{2}$ \\
\hline Age & $37.93 \pm 13.53$ & $40.76 \pm 15.67$ & $34.72 \pm 9.67$ & $5.14 * * *$ \\
\hline $\begin{array}{l}\text { Sex } \\
\text { Male } \\
\text { Female }\end{array}$ & $\begin{array}{l}303(59.6) \\
205(40.4)\end{array}$ & $\begin{array}{l}131(48.5) \\
139(51.5)\end{array}$ & $\begin{array}{l}172(72.3) \\
66(27.7)\end{array}$ & $29.64 * * *$ \\
\hline $\begin{array}{l}\text { Gender identity } \\
\text { Men } \\
\text { Women } \\
\text { Queer }\end{array}$ & $\begin{array}{l}289(56.9) \\
192(37.8) \\
26(5.1)\end{array}$ & $\begin{array}{l}125(46.3) \\
126(46.7) \\
19(7)\end{array}$ & $\begin{array}{l}164(68.9) \\
66(27.7) \\
7(2.9)\end{array}$ & $27.52 * * *$ \\
\hline $\begin{array}{l}\text { Sexual orientation } \\
\text { LG } \\
\text { Bisexual/Other }\end{array}$ & $\begin{array}{l}322(63.4) \\
186(36.6)\end{array}$ & $\begin{array}{l}178(65.9) \\
92(34.1)\end{array}$ & $\begin{array}{l}144(60.5) \\
94(39.5)\end{array}$ & 1.60 \\
\hline $\begin{array}{l}\text { Education level } \\
\leq \text { High school } \\
\geq \text { College }\end{array}$ & $\begin{array}{l}145(28.5) \\
362(71.3)\end{array}$ & $\begin{array}{l}104(38.5) \\
166(61.5)\end{array}$ & $\begin{array}{l}41(17.2) \\
196(82.4)\end{array}$ & $27.83 * * *$ \\
\hline $\begin{array}{l}\mathrm{SES}^{\mathrm{a}} \\
\text { Low-medium } \\
\text { High }\end{array}$ & $\begin{array}{l}447(88) \\
59(11.6)\end{array}$ & $\begin{array}{l}241(89.3) \\
29(10.7)\end{array}$ & $\begin{array}{l}206(86.6) \\
30(12.6)\end{array}$ & 0.47 \\
\hline
\end{tabular}

Note. *** $p<0.001$ Group differences in age were assessed using the Student's $t$ test for independent samples. Group differences in all other variables were assessed through the $\chi^{2}$ test

${ }^{a}$ Two Taiwanese participants were missing on this variable

LG $=$ Lesbian/Gay; SES = Socio-economic Status 
Nolen-Hoeksema, 2003), which is a 22 -item scale measuring the tendency to ruminate in response to feelings of depression and sadness (i.e., "How often do you think about how you do not seem to feel anything anymore"). Answers range from 1 (never/almost never) to 5 (always/almost always), with higher scores reflecting higher levels of rumination $(\alpha=0.99)$.

Resilience Resilience was measured using the Resilience Scale (Wagnild \& Young, 1993), which is a 10-item scale assessing resilience as a dynamic psychological process that buffers the impact of stress on health and promotes adjustment through both individual and group/community factors (i.e., "When I am in a difficult situation, I can usually find my way out of it"). Answers range from 1 (strongly agree) to 7 (strongly disagree), with higher scores reflecting higher resilience $(\alpha=0.93)$.

Health Health was assessed using five questions from a questionnaire designed to assess physical and mental health problems in the LGB+ population (D'Augelli, Grossman, Hershberger, \& O'Connell, 2001). One question concerns current overall mental health (i.e., "How would you describe your mental and emotional health at the present time?"), with answers ranging from 1 (excellent) to 5 (very poor). The other questions investigate changes in physical and mental health over the prior 5 years, with answers ranging from 1 (much better) to 5 (much worse) (i.e., How has your physical health changed in the past five years?). Higher scores reflect higher levels of health problems $(\alpha=0.93)$.

\section{Statistical Analyses}

Using Pearson's correlation coefficient, we calculated correlations between both distal and proximal minority stressors, rumination, resilience, and health, separately for the Italian and Taiwanese participants. Following this, we tested for statistically significant differences between countries using the Student's $t$ test. To test the relationships hypothesized in the moderated mediation model, we used a series of path analyses with 5000 bootstrap draws to determine the significance of the direct and indirect effects within the two national groups, using Mplus 8 (Muthén \& Muthén, 2017).

First, an unconditional model to determine the variance in health explained by discrimination was tested (model 1), followed by a conditional model (model 2) to examine the mediating role of ISS and rumination on the association between discrimination and health. Following this, the moderating effects of resilience on the associations between discrimination and ISS, rumination, and health were tested (model 3 ). Non-significant interactions were dropped (model 4) and the hypothesized moderated mediation analyses were tested. A series of Wald $\chi^{2}$ tests of parameter constraints were computed to identify nationality differences in all direct and indirect beta coefficients, with a significant Wald $\chi^{2}$ indicating that the coefficients were not equal and that differences existed. The model was adjusted for sexual orientation, biological sex, education, and SES.

\section{Results}

\section{Bivariate Correlations}

Bivariate correlations (Table 2 ) showed that minority stressors (i.e., discrimination and ISS) correlated positively with rumination and health problems, indicating that MS was associated with higher levels of distress. On the contrary, minority stressors correlated negatively with resilience, indicating that resilience was associated with lower levels of MS. The trend was the same in the Italian and Taiwanese participants.

\section{Differences among Italian and Taiwanese LGB+ Participants}

All differences between groups in the main variables were statistically significant: Italian participants resulted in higher discrimination and resilience, but lower ISS, rumination, and health problems than their Taiwanese counterparts (Table 3).

\section{Direct and Indirect Effects of Discrimination on Health}

A series of path analyses stratified by group nationality were used to test our hypotheses. All direct associations across the different path analysis models are reported in Table 4. With respect to Hypothesis 1, the findings indicated that discrimination was positively associated with health problems in both the Italian and the Taiwanese participants.

With regard to Hypothesis 2, we found that the association between discrimination and health was mediated by rumination, $B=-0.04, p=0.027,95 \% C I[-0.07,-0.01]$, but not ISS, $B=-0.02, p=0.081,95 \%$ CI $[-0.05,0.01]$, and only in the Italian participants. Specifically, higher levels of rumination increased the effect of discrimination on health problems in this group.

With respect to Hypothesis 3, we found that, only among Italian participants, resilience significantly moderated the relation between discrimination and ISS. Specifically, the association between discrimination and ISS was significant at 1 SD below the resilience mean, $B=0.26, p<0.001$, but not at 1 SD above the resilience mean, $B=-0.08, p=0.164$. In both national groups, the interaction effects of discrimination and resilience on rumination and health were not significant (Fig. 2).

Finally, with regard to Hypothesis 4 (Fig. 3), the results indicated that the indirect effect of discrimination on health via ISS was significant at $1 \mathrm{SD}$ below the resilience mean, $B=$ 
Table 2 Correlations Between Minority Stressors, Rumination, Resilience, and Health in Italian and Taiwanese LGB+ Participants

\begin{tabular}{llllll}
\hline Variables & 1 & 2 & 3 & 4 & 6 \\
\hline 1. Discrimination & - & $0.38^{* * *}$ & $0.29^{* * *}$ & $-0.35^{* * *}$ & $0.19^{* *}$ \\
2. ISS & $0.26^{* * *}$ & - & $0.21^{* *}$ & $-0.32^{* * *}$ & $-0.23^{* *}$ \\
3. Rumination & $0.39^{* * *}$ & $-0.44^{* * *}$ & - & $-0.30^{* * *}$ & $-0.21^{* *}$ \\
4. Resilience & $-0.31^{* * *}$ & $-0.49^{* * *}$ & $-0.57^{* * *}$ & - & $0.38^{* * *}$ \\
6. Health & $0.21^{* *}$ & $-0.41^{* * *}$ & $-0.40^{* * *}$ & $0.49^{* * *}$ & - \\
\hline
\end{tabular}

Note. $* * p<0.01 ; * * * p<0.001$. Italian participants scores are below diagonal and Taiwanese participants scores are above diagonal. ISS $=$ Internalized Sexual Stigma
$-0.04, p=0.038,95 \% C I[-0.08,-0.01]$, but not at $1 \mathrm{SD}$ above the resilience mean, $B=0.01, p=0.348,95 \% C I$ $[-0.01,0.04]$, and only in Italian participants. The model showed acceptable fit to the data for both the Italian, $\chi^{2}=$ $3.66, p=0.160, \mathrm{CFI}=0.99$, RMSEA $=0.056$ with $90 \%$ C.I. $[0.01,0.15]$, and the Taiwanese sample, $\chi^{2}=0.85, p=0.652$, $\mathrm{CFI}=1.00, \mathrm{RMSEA}=0.001$ with $90 \%$ C.I. $[0.01,0.11]$.

\section{Discussion}

The present study aimed at applying MST and the PMF to a group of Italian and Taiwanese LGB+ people, in order to explore potential differences between groups. The findings indicated that both MS levels and paths between variables differed between these national groups, depicting cultural diversity in MS-related experiences.

Specifically, Italian participants experienced higher discrimination and demonstrated higher resilience, but lower ISS, rumination, and health problems compared to their Taiwanese counterparts. ILGA-Europe's Annual Review of the Human Rights Situation of LGB+ people in Europe (ILGA-Europe, 2020) ranks Italy on it respective legal and policy practices for sexual and gender minority people (from 0 to $100 \%$ ) using criteria which includes family, hate crime and hate speech, discrimination, and legal gender recognition. Italy showed lower respect for human rights (23\%) than most European countries (i.e., $73 \%$ Belgium, $68 \%$ Denmark, $67 \%$
Spain, and 66\% UK). These data indicate that in Italy, LGB+ people still live in a society that supports discrimination and inequality for sexual and gender minorities. Nevertheless, the disadvantages experienced by the Italian LGB+ population may have stimulated them to create communities to oppose stigma and develop resilience skills (Baiocco et al., 2021). Indeed, though social and cultural climate can negatively affect LGB+ people's physical and mental health, such limitations have underscored a multitude of coping mechanisms and resilience for members of this often oppressed community. For these reasons, it is plausible to hypothesize that Italian people developed areas of strength resulting from such negative experiences: LGB+ people faced with adversity often develop significant strengths, resilience, and mechanisms for coping.

On the contrary, in Taiwan, in the past two decades, social acceptance toward LGB+ people has become widespread with an improvement in education and liberal values related to homosexuality and gender roles: Taiwan was the first Asian country (2019) to introduce same-sex marriage through voterinitiated referendums and allowed for same-sex second-parent adoption. Some recent studies (Lin et al.,2020; Ko et al., 2020) found that the increased exposure to same-sex marriage campaigns was associated with a high level of stress among LGB+ people in Taiwan. The referendums attracted people's attention and inspired nationwide debates on homosexuality and same-sex marriage: For most people in Taiwan, it was the first time that they discussed about homosexuality and same-

Table 3 Differences between Italian and Taiwanese LGB+ Participants on Minority Stressors, Rumination, Resilience, and Health

\begin{tabular}{|c|c|c|c|c|}
\hline Variables & $\begin{array}{l}\text { Total } \\
(n=508) \\
M \pm S D\end{array}$ & $\begin{array}{l}\text { Italian } \\
(n=270) \\
M \pm S D\end{array}$ & $\begin{array}{l}\text { Taiwanese } \\
(n=238) \\
M \pm S D\end{array}$ & $t$ \\
\hline Discrimination & $0.94 \pm 1.03$ & $1.05 \pm 1.10$ & $0.81 \pm 0.92$ & $2.70 * * *$ \\
\hline ISS & $1.94 \pm 0.85$ & $1.63 \pm 0.75$ & $2.28 \pm 0.83$ & $9.07 * * *$ \\
\hline Rumination & $48.52 \pm 15.29$ & $42.48 \pm 12.14$ & $56.19 \pm 15.47$ & $10.88 * * *$ \\
\hline Resilience & $2.97 \pm 0.58$ & $5.43 \pm 0.97$ & $5.01 \pm 1.05$ & $3.82 * * *$ \\
\hline Health & $3.17 \pm 0.75$ & $3.28 \pm .071$ & $3.02 \pm 0.77$ & $3.86^{* * *}$ \\
\hline
\end{tabular}

Note. $* * * p<0.001$. Group differences were assessed using the Student's $t$ test for independent samples. ISS = Internalized Sexual Stigma 


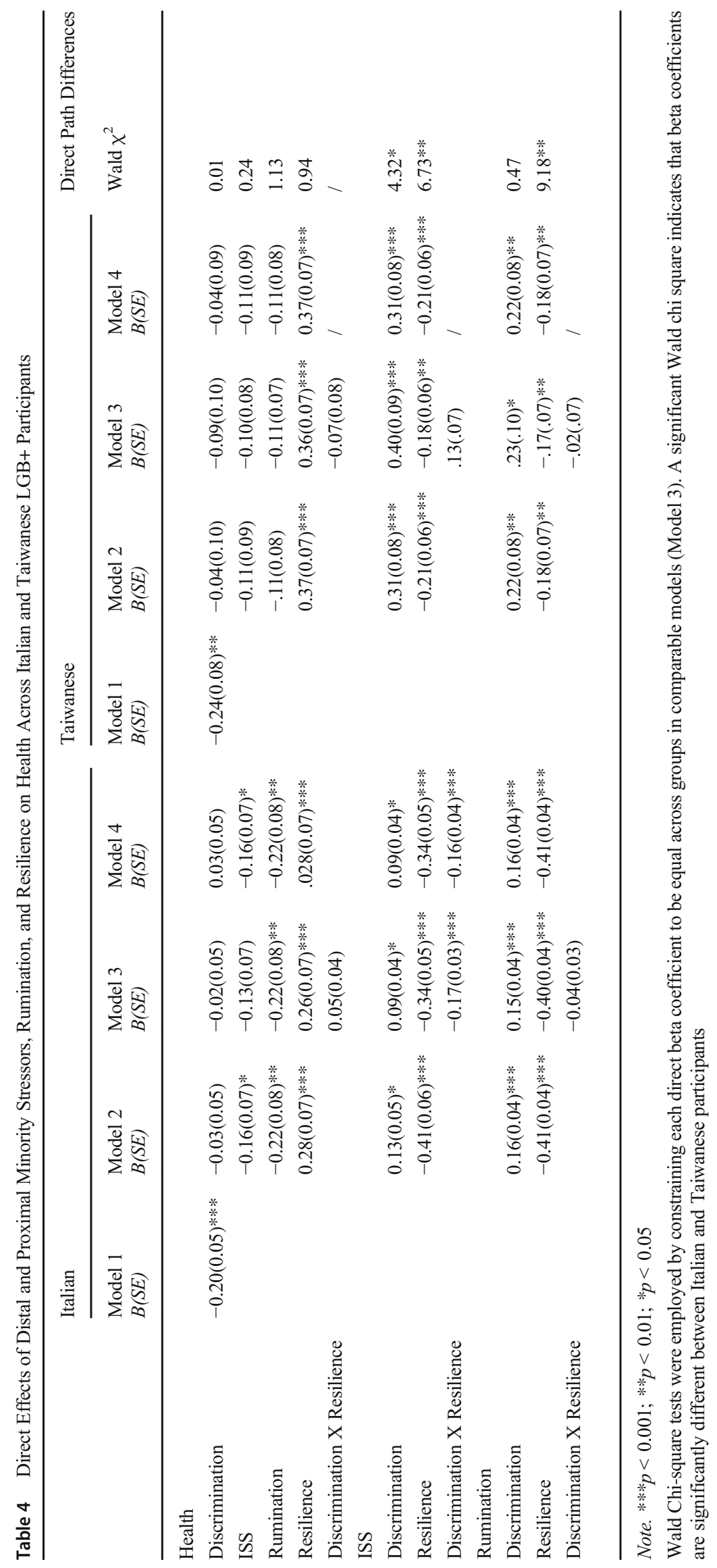


Fig. 2 Interaction Effect of Discrimination by Resilience on Internalized Sexual Stigma. Note: Low resilience $=1$ standard deviation below the mean; High resilience $=1$ standard deviation above the mean

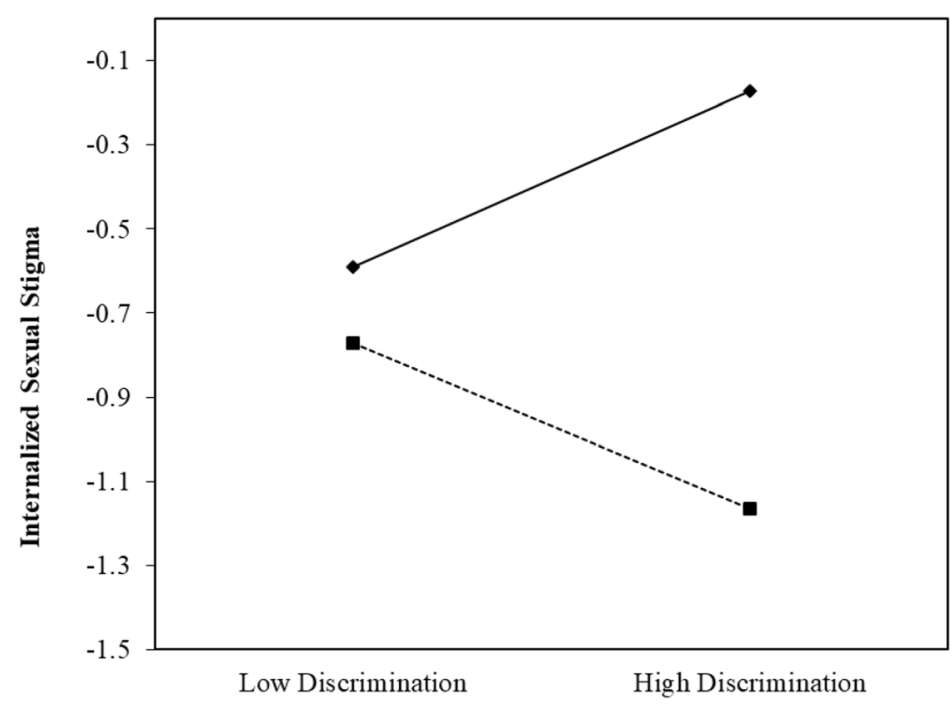

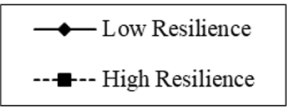

sex marriage (Ko et al., 2020). According to MST public debates on legalizing same-sex relationships may aggravate the negative social climate and interactions and thus negatively impact the daily lives and physical and mental health of LGB+ people. This social and cultural change in Taiwan in the last few years may explain the lower level of discrimination and the higher level of ISS, rumination, and health problems compared to their Italian counterparts.

The higher level of discrimination and resilience and the lower level of ISS, rumination, and health problems of Italian LGB+ people than their Taiwanese counterparts may also be explained by typical dynamics concerning identity visibility in $\mathrm{LGB}+$ people. Indeed, the literature highlights that greater visibility increases the likelihood that LGB+ people will experience stigmatizing episodes, leading them to reinforce their resilience strategies in order to protect themselves from the potentially negative effects of discrimination on their health
(Corlett, Di Marco, \& Arenas, 2019; Swank, Fahs, \& Frost, 2013; Watson \& Tatnell, 2019).

For instance, a particular form of visibility within the $\mathrm{LGB}+$ community is activism for LGB+ rights, which captures the engagement of $\mathrm{LGB}+$ people in actions aimed at promoting equality and rights within their group (Stürmer \& Simon, 2004). LGB+ activism increases subjects' risk of being discriminated due to the identity visibility it entails, even though it represents a health-enhancing response to MS (Frost, Fine, Torre, \& Cabana, 2019), associated with greater meaning in life and wellbeing (Klar \& Kasser, 2009; Thoits \& Hewitt, 2001). Thus, it seems plausible to hypothesize that the differences detected in the present study were due to the higher visibility of Italian LGB+ people compared to their Taiwanese counterparts. However, as we did not specifically measure this dimension, future studies should seek to validate our interpretative hypothesis about identity visibility by
Fig. 3 Interaction Effect of Discrimination by Resilience on Health via Internalized Sexual Stigma. Note: ISS = Internalized Sexual Stigma

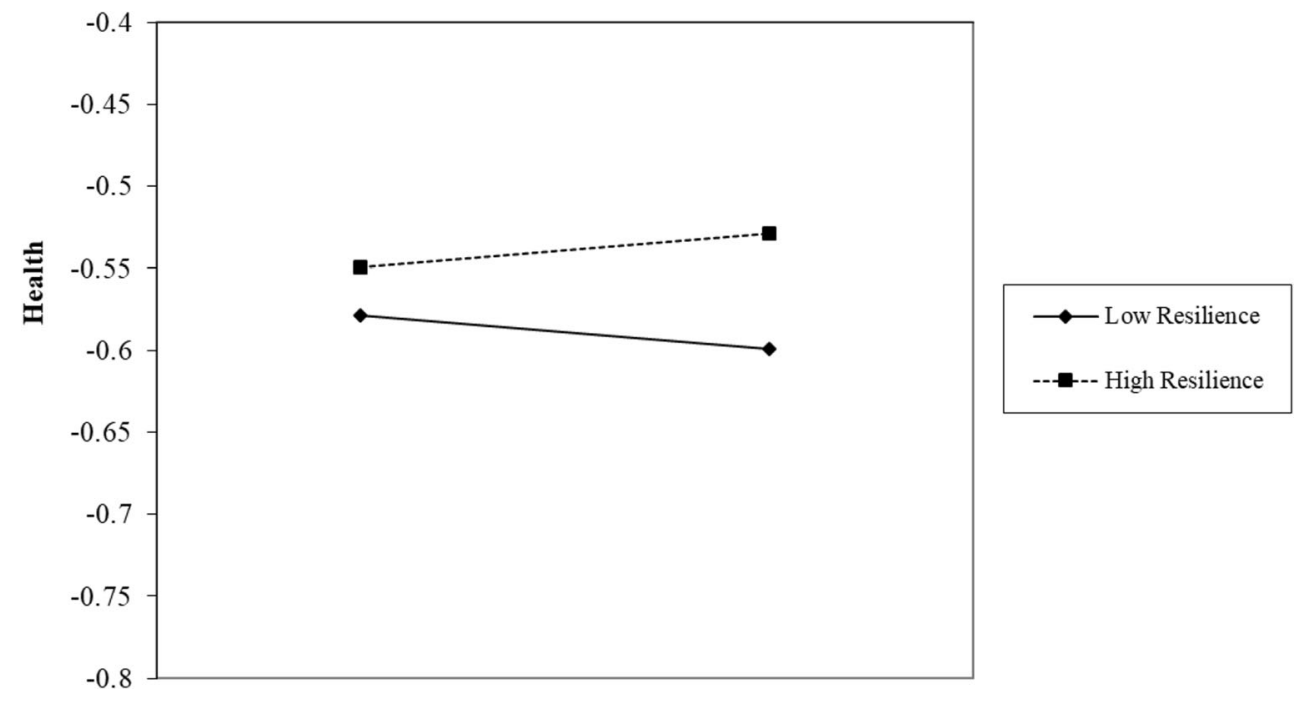


assessing the role of different forms of visibility (e.g., activism, coming out) in predicting MS and health problems in the LGB+ community.

Furthermore, the present results indicated that only the direct effect of discrimination on health problems was common among both the Italian and the Taiwanese participants, confirming Hypothesis 1. In contrast, the mediating role of ISS and rumination in the relationship between discrimination and health, as well as the moderating role of resilience as a factor protecting against the effect of discrimination on health, were partly significant only for the Italian group, thus partially confirming Hypotheses 2, 3, and 4.

These findings seem to indicate that the main path postulated by MST (i.e., discrimination-health) is valid, regardless of cultural differences. This confirms the robust foundation of MST, which postulates that distal stressors have a direct effect on health (Meyer, 2003). However, our results indicated that typical MST and PMF mediators (i.e., ISS, rumination) may differ across cultures. This may explain why discrimination led to health problems only in the Italian LGB+ participants. Likewise, even resilience did not seem capable of protecting Taiwanese LGB+ individuals from the negative effects of discrimination on health. This does not mean that MST and its clinical extension (i.e., the PMF) do not apply to Taiwanese LGB+ people. Rather, it seems more plausible to hypothesize that, in this particular Eastern culture, different mediators and moderators may be more relevant than those used in the current study. However, the role of resilience as a factor protecting Italian LGB+ people against the effect of stigma on health is noteworthy for two reasons. First, it is consistent with previous studies conducted in Western countries (e.g., Bariola et al., 2017; Jackson, 2017; Riggle et al., 2008). Second, it may inform psychological practice aimed at reducing stress and its negative impact on LGB+ people's mental health. Indeed, resilience may be reinforced through resilience-based psychological interventions, such as empowerment-based training group producing connectedness (e.g., Amodeo, Picariello, Valerio, \& Scandurra, 2018), LGB+ affirmative school-based interventions (e.g., Craig, 2013), minority stress-informed intervention (e.g., Heck, 2015), or affirmative counseling or individual psychotherapy (Scandurra, Mezza, Valerio, \& Vitelli, 2019).

Generally speaking, the Taiwanese and Italian cultures are significantly differentiated according to their respective collective versus individualistic attitudes. Although both cultures tend to stigmatize LGB+ people (Hu et al., 2019; Huang, Chan, \& Cui, 2020a; Huang, Luo, et al., 2020b; Picariello et al., 2019; Scandurra et al., 2020), their attitudinal differences may influence how the MS experienced by LGB+ people affects their health. Indeed, individualistic and collective cultures predict different individual efforts to achieve societal approval: Individualistic cultures emphasize individual achievement and uniqueness while collective cultures emphasize social connectedness and harmony (Sattler \& Lemke, 2019). This could suggest that, amongst Italian LGB+ people, mediators and moderators that explain why and how stigma influences them might be more individual, while amongst Taiwanese LGB+ persons, they might be more collective. Thus, in our Taiwanese participants, group resilience factors such as community belonging and social support may have acted as significant moderators, in contrast to the more individual resilience factors tested in the current study. To this end, future studies should use more comprehensive measures on protective factors, going beyond the individual level of resilience and embracing dimensions related to groupand community-level of resilience.

On the basis of our cultural considerations, future studies should seek to evaluate the relevance of other kinds of proximal stressors that could act as significant mediators in the relationship between discrimination and health in Taiwanese LGB+ people. To this end, MST postulates a continuum of proximal stressors, from the most subjective to the most relational, relating to concealment/disclosure of one's sexual identity or expectation of rejection (Meyer, 2003). Future studies should also aim at determining whether social psychological processes, such as social isolation (Eisenberg \& Resnick, 2006), could act as significant mediators in the relationship between discrimination and health in Taiwanese LGB+ people. Indeed, the PMF postulates that, in addition to emotion regulation processes, social/interpersonal processes may also act as mediators between stigma and health (Hatzenbuehler, 2009). Thus, future studies should seek to empirically test such hypotheses through culturally competent research that is able to effectively capture the cultural diversity of participants' experiences.

\section{Limitations of the Study}

The limitations of the present study include its cross-sectional nature, which did not enable secure inferences to be drawn about temporality and causality between variables. Second, the sample was not representative of either the general Italian or the general Taiwanese population of LGB+ people. Third, due to the relatively small sample size, it was not possible to explore potential differences between lesbian, gay, bisexual, and queer participants. Fourth, future studies should be conducted to consider people who are gender-expansive (such as persons that identify as genderqueer, gender nonconforming, agender, bigender, nonbinary, and so on).

\section{Conclusions}

This was the first study to compare the levels of and relationships between distal/proximal MS, rumination, resilience, and health among Italian and Taiwanese LGB+ people. The 
findings suggest that only the direct effect of discrimination on health was shared between the Italian and Taiwanese people, and not other typical MST and PMF mediators and moderators. The study highlights the need for further culturally sensitive research pertaining to the health of LGB+ people.

Acknowledgments The authors express their sincere gratitude to the LGB+ people who participated in this study. All authors who contributed significantly to the work have been identified.

Author Contributors R.B. designed and executed the study, assisted with the data analyses, and wrote the paper. F.R. and J.P. and collaborated with the design and writing of the study. V.B., and H. W. wrote part of the discussion. C.S. and S.I. collaborated with the design, analyzed the data and writing of the study. T.C. and R.B. collaborated in the writing and editing of the final manuscript. All authors read and approved the final manuscript.

Availability of Data and Material (Data Transparency) Data are available upon request in compliance with the General Data Protection Regulation (GDPR; Regulation EU2016/679) on the protection of natural persons with regard to the processing of personal data and on the free movement of such data.

Funding Open Access funding provided by Università degli Studi di Roma La Sapienza. For Salvatore Ioverno, part of the administrative support for this research was provided by the Research Foundation Flanders (Fonds voor Wetenschappelijk Onderzoek; FWO), [grant number $12 \mathrm{~V} 8120 \mathrm{~N}]$. For Tesheng Chang, this research was supported by the Research Foundation-Ministry of Science and Technology [grant number MOST-108-2918-I-259-001]. All authors who contributed significantly to the work have been identified.

\section{Compliance with Ethical Standards}

Conflicts of Interest/Competing Interests No competing financial interests exist.

Ethics Approval All procedures performed in studies involving human participants were conducted in accordance with the ethical standards of the institutional and national research committee and with the 2013 Declaration of Helsinki and its later amendments or comparable ethical standards. This article does not refer to any studies with animals performed by any of the authors. The responsibility of data analysis and elaboration should be ascribed to the authors themselves, who autonomously conceived the research strategy.

Informed Consent Informed consent was obtained from all individual participants included in the study.

Open Access This article is licensed under a Creative Commons Attribution 4.0 International License, which permits use, sharing, adaptation, distribution and reproduction in any medium or format, as long as you give appropriate credit to the original author(s) and the source, provide a link to the Creative Commons licence, and indicate if changes were made. The images or other third party material in this article are included in the article's Creative Commons licence, unless indicated otherwise in a credit line to the material. If material is not included in the article's Creative Commons licence and your intended use is not permitted by statutory regulation or exceeds the permitted use, you will need to obtain permission directly from the copyright holder. To view a copy of this licence, visit http://creativecommons.org/licenses/by/4.0/.

\section{References}

Amodeo, A. L., Picariello, S., Valerio, P., \& Scandurra, C. (2018). Empowering transgender youths: Promoting resilience through a group training program. Journal of Gay \& Lesbian Mental Health, 22(1), 3-19.

Baiocco, R., \& Pistella, J. (2019). "Be as you are" clinical research center at the Sapienza University of Rome. Journal of Gay \& Lesbian Mental Health, 23(4), 376-379.

Baiocco, R., Pezzella, A., Pistella, J. ... \& Papadopulous, I. (2021). LGBT+ training needs for health and social care professionals: A cross-cultural comparison among seven European countries. Sexuality Research and Social Policy. Advance online publication. https://doi.org/10.1007/s13178-020-00521-2.

Balsam, K. F., Beadnell, B., \& Molina, Y. (2013). The daily heterosexist experiences questionnaire: Measuring minority stress among lesbian, gay, bisexual, and transgender adults. Measurement and Evaluation in Counseling and Development, 46(1), 3-25.

Bariola, E., Lyons, A., \& Lucke, J. (2017). Flourishing among sexual minority individuals: Application of the dual continuum model of mental health in a sample of lesbians and gay men. Psychology of Sexual Orientation and Gender Diversity, 4(1), 43-53.

Berg, R. C., Munthe-Kaas, H. M., \& Ross, M. W. (2016). Internalized homonegativity: A systematic mapping review of empirical research. Journal of Homosexuality, 63(4), 541-558.

Bostwick, W. B., Boyd, C. J., Hughes, T. L., West, B. T., \& McCabe, S. E. (2014). Discrimination and mental health among lesbian, gay, and bisexual adults in the United States. American Journal of Orthopsychiatry, 84(1), 35-45.

Corlett, S., Di Marco, D., \& Arenas, A. (2019). 'Coming out' across cultures: Examining the experiences of Ecuadorian and Spanish LGB employees. Current Psychology. Advance online publication. https://doi.org/10.1007/s12144-019-00463-y.

Craig, S. L. (2013). Affirmative supportive safe and empowering talk (ASSET): Leveraging the strengths and resiliencies of sexual minority youth in school-based groups. Journal of LGBT Issues in Counseling, 7(4), 372-386.

D’Augelli, A. R., Grossman, A. H., Hershberger, S. L., \& O'Connell, T. S. (2001). Aspects of mental health among older lesbian, gay, and bisexual adults. Aging \& Mental Health, 5(2), 149-158.

de Lira, A. N., \& de Morais, N. A. (2018). Resilience in lesbian, gay, and bisexual (LGB) populations: An integrative literature review. Sexuality Research and Social Policy, 15(3), 272-282.

Denton, F. N., Rostosky, S. S., \& Danner, F. (2014). Stigma-related stressors, coping self-efficacy, and physical health in lesbian, gay, and bisexual individuals. Journal of Counseling Psychology, 61(3), 383-391.

Douglass, R, P., \& Conlin, S, E. (2020). Minority stress among LGB people: Investigating relations among distal and proximal stressors. Current Psychology. Advance online publication. https://doi.org/10. 1007/s12144-020-00885-z.

Eisenberg, M. E., \& Resnick, M. D. (2006). Suicidality among gay, lesbian and bisexual youth: The role of protective factors. Journal of Adolescent Health, 39(5), 662-668.

Frost, D. M., Fine, M., Torre, M. E., \& Cabana, A. (2019). Minority stress, activism, and health in the context of economic precarity: Results from a national participatory action survey of lesbian, gay, bisexual, transgender, queer, and gender non-conforming youth. American Journal of Community Psychology, 63(3-4), 511-526.

Gonzales, G., \& Henning-Smith, C. (2017). Health disparities by sexual orientation: Results and implications from the behavioral risk factor surveillance system. Journal of Community Health, 42(6), 1163 1172.

Hässler, T., Ullrich, J., Bernardino, M., Shnabel, N., Van Laar, C., Valdenegro, D., et al. (2020). A large-scale test of the link between 
intergroup contact and support for social change. Nature Human Behaviour, 4(4), 380-386.

Hatzenbuehler, M. L. (2009). How does sexual minority stigma "get under the skin"? A psychological mediation framework. Psychological Bulletin, 135(5), 707-730.

Hatzenbuehler, M. L. (2010). Social factors as determinants of mental health disparities in LGB populations: Implications for public policy. Social Issues and Policy Review, 4(1), 31-62.

Hatzenbuehler, M. L., Dovidio, J. F., Nolen-Hoeksema, S., \& Phills, C. E. (2009). An implicit measure of anti-gay attitudes: Prospective associations with emotion regulation strategies and psychological distress. Journal of Experimental Social Psychology, 45(6), 13161320.

Heck, N. C. (2015). The potential to promote resilience: Piloting a minority stress-informed, GSA-based, mental health promotion program for LGBTQ youth. Psychology of Sexual Orientation and Gender Diversity, 2(3), 225-231.

Hill, C. A., \& Gunderson, C. J. (2015). Resilience of lesbian, gay, and bisexual individuals in relation to social environment, personal characteristics, and emotion regulation strategies. Psychology of Sexual Orientation and Gender Diversity, 2(3), 232-252.

Hu, H. F., Chang, Y. P., Lin, C., \& Yen, C. F. (2019). Quality of life of gay and bisexual men during emerging adulthood in Taiwan: Roles of traditional and cyber harassment victimization. PLoS One, 14(2), e0213015.

Huang, Y. T., Chan, R. C. H., \& Cui, L. (2020a). Filial piety, internalized homonegativity, and depressive symptoms among Taiwanese gay and bisexual men: A mediation analysis. American Journal of Orthopsychiatry, 90(3), 340-349.

Huang, Y. T., Luo, H., Ko, N. Y., \& Yen, C. F. (2020b). Perceived attitudes toward lesbian, gay, and bisexual (LGB) issues and mental health among Taiwanese LGB adults: The mediating role of selfacceptance. Archives of Sexual Behavior, 49, 1671-1682. https:// doi.org/10.1007/s10508-020-01686-y.

ILGA-Europe. (2020). Annual review of the human rights situation of lesbian, gay, bisexual, trans and intersex people covering events that occurred in Europe and Central Asia between JanuaryDecember 2019. Brussels: ILGA-Europe.

Jackson, S. D. (2017). Connection is the antidote: Psychological distress, emotional processing, and virtual community building among LGBTQ students after the Orlando shooting. Psychology of Sexual Orientation and Gender Diversity, 4(2), 160-168.

Kiekens, W., la Roi, C., Bos, H. M., Kretschmer, T., van Bergen, D. D., \& Veenstra, R. (2020). Explaining health disparities between heterosexual and LGB adolescents by integrating the minority stress and psychological mediation frameworks: Findings from the TRAILS study. Journal of Youth and Adolescence, 49, 1767-1782. https:// doi.org/10.1007/s10964-020-01206-0.

Klar, M., \& Kasser, T. (2009). Some benefits of being an activist: Measuring activism and its role in psychological well-being. Political Psychology, 30(5), 755-777.

Ko, N. Y., Lin, I., Huang, Y. T., Chen, M. H., Lu, W. H., \& Yen, C. F. (2020). Associations of perceived socially unfavorable attitudes toward homosexuality and same-sex marriage with suicidal ideation in Taiwanese people before and after same-sex marriage referendums. International Journal of Environmental Research and Public Health, 17(3), 1047.

Kwon, P. (2013). Resilience in lesbian, gay, and bisexual individuals. Personality and Social Psychology Review, 17(4), 371-383.

Lewis, R. J., Milletich, R. J., Mason, T. B., \& Derlega, V. J. (2014). Pathways connecting sexual minority stressors and psychological distress among lesbian women. Journal of Gay \& Lesbian Social Services, 26(2), 147-167.

Lin, H. C., Chen, Y. L., Ko, N. Y., Chang, Y. P., Lu, W. H., \& Yen, C. F. (2020). Impacts of public debates on legalizing the same-sex relationships on People's daily lives and their related factors in Taiwan.
International Journal of Environmental Research and Public Health, 17(22), 8606

Lingiardi, V., Baiocco, R., \& Nardelli, N. (2012). Measure of internalized sexual stigma for lesbians and gay men: A new scale. Journal of Homosexuality, 59(8), 1191-1210.

Lingiardi, V., Nardelli, N., Ioverno, S., Falanga, S., Di Chiacchio, C., et al. (2016). Homonegativity in Italy: Cultural issues, personality characteristics, and demographic correlates with negative attitudes toward lesbians and gay men. Sexuality Research and Social Policy, 13(2), 95-108.

Liu, H., Feng, T., Ha, T., Liu, H., Cai, Y., Liu, X., \& Li, J. (2011). Chinese culture, homosexuality stigma, social support and condom use: A path analytic model. Stigma Research and Action, 1(1), 27 35.

Luthar, S. S., Cicchetti, D., \& Becker, B. (2000). The construct of resilience: A critical evaluation and guidelines for future work. Child Development, 71(3), 543-562.

Matsuno, E., \& Israel, T. (2018). Psychological interventions promoting resilience among transgender individuals: Transgender resilience intervention model (TRIM). The Counseling Psychologist, 46(5), $632-655$

McLaughlin, K. A., \& Hatzenbuehler, M. L. (2009). Mechanisms linking stressful life events and mental health problems in a prospective, community-based sample of adolescents. Journal of Adolescent Health, 44(2), 153-160.

Meyer, I. H. (2003). Prejudice, social stress, and mental health in lesbian, gay, and bisexual populations: Conceptual issues and research evidence. Psychological Bulletin, 129(5), 674-697.

Meyer, I. H. (2015). Resilience in the study of minority stress and health of sexual and gender minorities. Psychology of Sexual Orientation and Gender Diversity, 2(3), 209-213.

Muthén, L. K., \& Muthén, B. O. (2017). Mplus 'user's guide: Statistical analysis with latent variables, 'user's guide. Los Angeles, CA: Muthén \& Muthén.

Petrocchi, N., Pistella, J., Salvati, M., Carone, N., Laghi, F., \& Baiocco, R. (2020). I embrace my LGB identity: Self-reassurance, social safeness, and the distinctive relevance of authenticity to well-being in Italian lesbians, gay men, and bisexual people. Sexuality Research and Social Policy, 17(1), 75-86.

Picariello, S., Scandurra, C., Schwartz, S. J., \& Amodeo, A. L. (2019). Identity complexity and integration in lesbian, gay, bisexual and heterosexual adolescents and emerging adults: Implications for clinical practice. Mediterranean Journal of Clinical Psychology, 7(2), $1-25$.

Potter, E. C., \& Patterson, C. J. (2019). Health-related quality of life among lesbian, gay, and bisexual adults: The burden of health disparities in 2016 behavioral risk factor surveillance system data. LGBT Health, 6(7), 357-369.

Riggle, E. D. B., Whitman, J. S., Olson, A., Rostosky, S. S., \& Strong, S. (2008). The positive aspects of being a lesbian or gay man. Professional Psychology: Research and Practice, 39(2), 210-217.

Russell, S. T., \& Fish, J. N. (2016). Mental health in lesbian, gay, bisexual, and transgender (LGBT) youth. Annual Review of Clinical Psychology, 12, 465-487. https://doi.org/10.1146/annurev-clinpsy021815-093153.

Salvati, M., Pistella, J., Ioverno, S., Laghi, F., \& Baiocco, R. (2018). Coming out to siblings and internalized sexual stigma: The moderating role of gender in a sample of Italian participants. Journal of GLBT Family Studies, 14(5), 405-424.

Sattler, F. A., \& Lemke, R. (2019). Testing the cross-cultural robustness of the minority stress model in gay and bisexual men. Journal of Homosexuality, 66(2), 189-208.

Scandurra, C., Mezza, F., Valerio, P., \& Vitelli, R. (2019). Approcci affermativi e rilevanza del minority stress nel counseling psicologico con persone LGBT: Una revisione della letteratura internazionale [affirmative approaches and relevance of minority stress in 
psychological counseling with LGBT people: A review of the international literature]. Psicoterapia e Scienze Umane, 53(1), 67-92.

Scandurra, C., Pennasilico, A., Esposito, C., Mezza, F., Vitelli, R., Bochicchio, V., Maldonato, N. M., \& Amodeo, A. L. (2020). Minority stress and mental health in Italian bisexual people. Social Sciences, 9(4), 46.

Stürmer, S., \& Simon, B. (2004). The role of collective identification in social movement participation: A panel study in the context of the German gay movement. Personality and Social Psychology Bulletin, 30(3), 263-277.

Swank, E., Fahs, B., \& Frost, D. M. (2013). Region, social identities, and disclosure practices as predictors of heterosexist discrimination against sexual minorities in the United States. Sociological Inquiry, 83(2), 238-258.

Szymanski, D. M., Dunn, T. L., \& Ikizler, A. S. (2014). Multiple minority stressors and psychological distress among sexual minority women: The roles of rumination and maladaptive coping. Psychology of Sexual Orientation and Gender Diversity, 1(4), 412-421.

Thoits, P. A., \& Hewitt, L. N. (2001). Volunteer work and well-being. Journal of Health and Social Behavior, 42(2), 115-131.

Timmins, L., Rimes, K. A., \& Rahman, Q. (2020). Minority stressors, rumination, and psychological distress in lesbian, gay, and bisexual individuals. Archives of Sexual Behavior, 49(2), 661-680.
Toomey, R. B., Ryan, C., Diaz, R. M., \& Russell, S. T. (2018). Coping with sexual orientation-related minority stress. Journal of Homosexuality, 65(4), 484-500.

Treynor, W., Gonzalez, R., \& Nolen-Hoeksema, S. (2003). Rumination reconsidered: A psychometric analysis. Cognitive Therapy and Research, 27(3), 247-259.

Wagnild, G. M., \& Young, H. M. (1993). Development and psychometric evaluation of the resilience scale. Journal of Nursing Measurement. l(2), 165-178.

Walch, S. E., Ngamake, S. T., Bovornusvakool, W., \& Walker, S. V. (2016). Discrimination, internalized homophobia, and concealment in sexual minority physical and mental health. Psychology of Sexual Orientation and Gender Diversity, 3(1), 37-48.

Watson, C., \& Tatnell, R. (2019). Resilience and non-suicidal self-injury in LGBTQIA+ people: Targets for prevention and intervention. Current Psychology. Advance online publication. https://doi.org/ 10.1007/s12144-019-00573-7.

Publisher's Note Springer Nature remains neutral with regard to jurisdictional claims in published maps and institutional affiliations. 